\title{
Determining Inference Semantics for Disjunctive Logic Programs (Extended Abstract)*
}

\author{
Yi-Dong Shen ${ }^{1}$ and Thomas Eiter $^{2}$ \\ ${ }^{1}$ State Key Laboratory of Computer Science, Institute of Software, Chinese Academy of Sciences, \\ Beijing, China \\ ${ }^{2}$ Institut of Logic and Computation, Technische Universität Wien, Favoritenstraße 9-11, A-1040 Vienna, \\ Austria \\ ydshen@ios.ac.cn, eiter@kr.tuwien.ac.at
}

\begin{abstract}
[Gelfond and Lifschitz, 1991] introduced simple disjunctive logic programs and defined the answer set semantics called GL-semantics. We observed that the requirement of GL-semantics, i.e., an answer set should be a minimal model of the GLreduct may be too strong and exclude some answer sets that would be reasonably acceptable. To address this, we present a novel and more permissive semantics, called determining inference semantics.
\end{abstract}

\section{Introduction}

In a seminal paper, [Gelfond and Lifschitz, 1991] introduced simple disjunctive logic programs, where in rule heads the disjunction operator "|" is used to express incomplete information, and defined the answer set semantics (called $G L$ semantics) based on a program transformation (called $G L$ reduct) and the minimal model requirement. Our observations reveal that the requirement of GL-semantics, i.e., an answer set should be a minimal model of rules of the GL-reduct, may sometimes be too strong and exclude some answer sets that would be reasonably acceptable, as illustrated in the following example.

Example 1. Consider the simple disjunctive program.

$$
\begin{aligned}
\Pi: & a \mid b \\
& b \leftarrow a \\
& c \leftarrow a \\
& c \leftarrow \neg c
\end{aligned}
$$

Intuitively, rule (1) presents two alternatives for answer set construction, namely $a$ or $b$, and rules (2) and (3) infer $b$ and $c$, respectively if $a$ has already been derived. Rule (4) is a constraint stating that there is no answer set that does not contain $c$. We distinguish between the following two cases. Suppose that we choose $a$ from rule (1); then by rules (2) and (3) we obtain a potential answer set $I_{1}=\{a, b, c\}$. $I_{1}$ satisfies the constraint (4), so it is a candidate answer set for $\Pi$. Alternatively, suppose that we choose $b$ from rule (1). As $a$ is not inferred from rule (1), rules (2) and (3) are not applicable;

\footnotetext{
${ }^{*}$ This paper is an extended abstract of the article [Shen and Eiter, 2019] in Artificial Intelligence, 277:1-28, 2019.
}

so rules (1), (2) and (3) together infer a potential answer set $I_{2}=\{b\}$. As $I_{2}$ does not satisfy the constraint (4), it is not a candidate answer set for $\Pi$. Consequently, $I_{1}=\{a, b, c\}$ is a minimal candidate answer set and thus we expect it to be an answer set of $\Pi$. However, $I_{1}$ is not an answer set under GL-semantics because it is not a minimal model of $\Pi$.

To address this, we present a more permissive semantics:

(1) We present a general answer set semantics for disjunctive programs, called determining inference semantics (DI-semantics for short), which interprets the operator $\mid$ in rule heads differently from the classical connective $V$, and does not require that answer sets should be minimal models. Specifically, we formalize the rule head operator | by introducing a head selection function sel, i.e., for every interpretation $I$ and rule head $H_{1}|\cdots| H_{k}, \operatorname{sel}\left(H_{1}|\cdots| H_{k}, I\right)$ nondeterministically selects one alternative $H_{i}$ satisfied by $I$. Then we define answer sets as follows: (i) Given an interpretation $I$ and a selection function sel, we transform a disjunctive program $\Pi$ into a normal program $\Pi_{\text {sel }}^{I}$, called disjunctive program reduct, such that for every rule head $(r) \leftarrow \operatorname{body}(r)$ in $\Pi$, $\operatorname{sel}(\operatorname{head}(r), I) \leftarrow \operatorname{body}(r)$ is in $\Pi_{\text {sel }}^{I}$ if $I$ satisfies $\operatorname{body}(r)$; (ii) given a base answer set semantics $\mathcal{X}$ for normal programs, we define $I$ to be a candidate answer set w.r.t. $\mathcal{X}$ if $I$ is an answer set of $\Pi_{\text {sel }}^{I}$ under $\mathcal{X}$; and (iii) we define $I$ to be an answer set w.r.t. $\mathcal{X}$ if $I$ is a minimal candidate answer set. Such answer sets are called DI-answer sets.

(2) By replacing the base semantics $\mathcal{X}$ in the above general semantics with the $\mathrm{GL}_{n l p}$-semantics defined by [Gelfond and Lifschitz, 1988], we induce a DI-semantics for simple disjunctive programs (definitions follow below). We show that an answer set under GL-semantics is an answer set under DI-semantics, but not vice versa; the main reason behind is that GL-semantics interprets the operator | in rule heads as the classical connective $\vee$ and further requires that answer sets must be minimal models; this may exclude some desired answer sets. To clearly see the essential difference of DIsemantics from GL-semantics, we also present a new characterization of GL-semantics in terms of a disjunctive program reduct $\Pi_{\text {sel }}^{I}$. Based on this characterization, we obtain a satisfactory solution to an open problem of [Hitzler and Seda, 1999], which was to characterize split normal derivatives of a simple disjunctive program $\Pi$.

(3) By replacing the base semantics $\mathcal{X}$ with the well- 
justified semantics defined by [Shen et al., 2014], we further induce a DI-semantics for general disjunctive programs consisting of rules of the form $H_{1}|\cdots| H_{k} \leftarrow B$, where $B$ and every $H_{i}$ are arbitrary first-order formulas. This closes the open issue of [Shen et al., 2014] how to extend the welljustified semantics from general normal programs with rules of the form $H_{1} \leftarrow B$ to general disjunctive programs.

(4) Finally, we show that in the propositional case deciding whether a simple disjunctive program $\Pi$ has some DIanswer set is NP-complete, and deciding whether a ground literal is true in some (resp. every) DI-answer set of $\Pi$ is $\Sigma_{2}^{p}$-complete (resp. $\Pi_{2}^{p}$-complete). This is in contrast to GLsemantics, where deciding whether a simple disjunctive program has GL-answer sets is $\Sigma_{2}^{p}$-complete [Eiter and Gottlob, 1995]. For general disjunctive programs, the complexity of DI-semantics increases to $\Sigma_{2}^{p}$-completeness for DI-answer set existence and to $\Sigma_{3}^{p}$-completeness and $\Pi_{3}^{p}$-completeness for brave and cautious reasoning, respectively.

For an extensive discussion of historical and philosophical background, we refer to [Shen and Eiter, 2019].

\section{Disjunctive Programs}

We take a first-order logic language $\mathcal{L}_{\Sigma}$ with equality. A firstorder theory (or theory) is a set $T$ of closed formulas. By $\mathcal{N}_{\Sigma}$ we denote the set of all ground (variable-free) terms of $\Sigma$, and by $\mathcal{H}_{\Sigma}$ the set of all ground atoms. An interpretation $I$ is a subset of $\mathcal{H}_{\Sigma}$ such that for any ground atom $A, I$ satisfies $A$ if $A \in I$, and $\neg A$ if $A \notin I$. The notion of satisfaction/models of a formula/theory in $I$ is defined as usual. A theory $T$ entails a closed formula $F$, denoted $T \models F$, if all models of $T$ are models of $F$. For an interpretation $I$, we let $I^{-}=\mathcal{H}_{\Sigma} \backslash I$ and $\neg I^{-}=\left\{\neg A \mid A \in I^{-}\right\}$.

Definition 1. A general disjunctive program (disjunctive program for short) is a finite set of rules of the form

$$
H_{1}|\cdots| H_{k} \leftarrow B
$$

where $k>0$, and $B$ and the $H_{i}$ 's are first-order formulas.

For a rule $r$, we refer to $B$ and $H_{1}|\cdots| H_{k}$ as its body and head, denoted $\operatorname{body}(r)$ and $h e a d(r)$, respectively. We also refer to each $H_{i}$ as a head formula. A constraint is a rule of the form $\perp \leftarrow B$. A rule $A \leftarrow \neg A$ amounts to a constraint $\perp \leftarrow \neg A$. A disjunctive program is a general normal program (normal program for short) if $k=1$ for every rule; a simple disjunctive program if each $H_{i}$ is an atom and $B$ is a conjunction of literals, and a simple normal program if additionally $k=1$. A positive simple normal/disjunctive program is a simple normal/disjunctive program without negative literals. The grounding of a disjunctive program $\Pi$, obtained by substituting the free variables in $\Pi$ with constants in all possible ways, is denoted ground $(\Pi)$.

An interpretation $I$ satisfies a rule head $H_{1}|\cdots| H_{k}$ if it satisfies some $H_{i}$; $I$ satisfies a rule $r$ if it either satisfies $h e a d(r)$ or it does not satisfy body $(r) ; I$ is a model of a disjunctive program $\Pi$ if $I$ satisfies every rule $r \in \operatorname{ground}(\Pi)$.

Let $\Pi$ be a simple disjunctive program and $I$ an interpretation. The GL-reduct of $\Pi$ w.r.t. $I$, written as $\Pi^{I}$, is obtained from ground $(\Pi)$ by (1) removing all rules whose bodies contain some $\neg C_{i}$ with $C_{i} \in I$, and (2) removing from the remaining rules all $\neg C_{i}$. The $G L$-semantics defines $I$ to be an answer set of $\Pi$ (referred to as GL-answer set) if $I$ is a minimal model of $\Pi^{I}$ [Gelfond and Lifschitz, 1991]. When $\Pi$ is a simple normal program, the $G L_{n l p}$-semantics defines $I$ to be an answer set of $\Pi$ if $I$ is the least model of $\Pi^{I}$. For simple normal programs, GL- and $\mathrm{GL}_{n l p}$-semantics coincide.

Remark 1. If we replace $\mid$ with $\vee$ in rule heads and let $\Pi_{\vee}^{I}$ be $\Pi^{I}$ with all occurrences of $\mid$ replaced by $\vee$, then $\Pi^{I}$ has the same minimal models as $\Pi_{\vee}^{I}$. Thus $I$ is an answer set of $\Pi$ under GL-semantics iff $I$ is a minimal model of $\Pi^{I}$ iff $I$ is a minimal model of $\Pi_{\vee}^{I}$. This means that in GL-semantics the use of $\mid$ in rule heads amounts in essence to disjunction $\vee$.

\section{Determining Inference (DI) Semantics}

Under the constructive view of the operator | as a nondeterministic inference operator, every rule head $\mathcal{H}=H_{1}$ ... $H_{k}$ in a disjunctive program can be viewed as a set $\left\{H_{1}, \cdots, H_{k}\right\}$ of alternatives. As these alternatives may have different variants (i.e., every $H_{i}$ can be expressed as different yet logically equivalent formulas) and appear in different orders in rule heads, we introduce a notion of variant rule heads.

Definition 2. Rule heads $\mathcal{H}_{1}=E_{1}|\cdots| E_{k}$ and $\mathcal{H}_{2}=$ $F_{1}|\cdots| F_{l}$, where the $E_{i}$ 's and $F_{j}$ 's are closed formulas, are variant rule heads if for every $E_{i}$ in $\mathcal{H}_{1}$ some $F_{j}$ in $\mathcal{H}_{2}$ exists with $E_{i} \equiv F_{j}$, and vice versa for every $F_{j}$ in $\mathcal{H}_{2}$ some $E_{i}$ in $\mathcal{H}_{1}$ exists with $E_{i} \equiv F_{j}$.

Intuitively, variant rule heads $\mathcal{H}_{1}$ and $\mathcal{H}_{2}$ represent the same set of alternatives and should be treated the same. If $k>l$, then $\mathcal{H}_{1}$ must have some head formulas that are logically equivalent. Moreover, rule heads in a simple disjunctive program are variant rule heads iff they have the same atoms.

Definition 3. Let $\Pi$ be a disjunctive program and $\mathcal{I}$ the collection of all interpretations. Let $\mathcal{H} \mathcal{D}_{\Pi}$ be the set of all rule heads in ground $(\Pi)$, and $\mathcal{H} \mathcal{F}_{\Pi}$ the set of all head formulas in $\mathcal{H} \mathcal{D}_{\Pi}$. A head selection for $\Pi$ is a function sel : $\mathcal{H} \mathcal{D}_{\Pi} \times \mathcal{I} \rightarrow$ $\mathcal{H} \mathcal{F}_{\Pi} \cup\{\perp\}$ such that for every interpretation $I \in \mathcal{I}$ and every rule $r \in \operatorname{ground}(\Pi)$,

$\operatorname{sel}(\operatorname{head}(r), I)= \begin{cases}F_{i}, & \text { if head }(r) \text { has some head formula } \\ & F_{i} \text { that is satisfied by } I \\ \perp, & \text { otherwise, }\end{cases}$

such that for every variant rule heads $\mathcal{H}_{1}$ and $\mathcal{H}_{2}$ in $\mathcal{H} \mathcal{D}_{\Pi}$, $\operatorname{sel}\left(\mathcal{H}_{1}, I\right) \equiv \operatorname{sel}\left(\mathcal{H}_{2}, I\right)$.

A head selection function sel formalizes the operator as a nondeterministic operator; i.e., for any interpretation $I$, $\operatorname{sel}\left(F_{1}|\cdots| F_{k}, I\right)$ returns from a rule head $F_{1}|\cdots| F_{k}$ one of the alternatives $F_{i}$ satisfied by $I$, or it returns $\perp$ if there is no $F_{i}$ that is satisfied by $I$. For variant rule heads, it returns logically equivalent alternatives that are satisfied by $I$.

Definition 4. Let $\Pi$ be a disjunctive program, $I$ an interpretation and sel a head selection function. The reduct of $\Pi$ w.r.t. $I$ and sel is $\Pi_{\text {sel }}^{I}=\{\operatorname{sel}(h e a d(r), I) \leftarrow \operatorname{bod} y(r) \mid r \in$ ground $(\Pi)$ s.t. $I$ satisfies body $(r)\}$.

A reduct $\Pi_{\text {sel }}^{I}$ is a normal program; therefore we can apply any existing answer set semantics for normal programs to compute answer sets of $\Pi_{\text {sel }}^{I}$. Intuitively $I$ is a candidate answer set of $\Pi$ if $I$ is an answer set of $\Pi_{\text {sel }}^{I}$, and $I$ is an answer set of $\Pi$ if $I$ is minimal among all candidate answer sets. 
Definition 5. Let $I$ be a model of a disjunctive program $\Pi$, and $\mathcal{X}$ be an answer set semantics for normal programs. Then $I$ is an answer set of $\Pi$ w.r.t. $\mathcal{X}$ if (1) for some head selection function sel, $I$ is an answer set of $\Pi_{\text {sel }}^{I}$ under $\mathcal{X}$, and (2) $\Pi$ has no model $J \subset I$ satisfying condition (1).

Due to the use of head selection functions, the above semantics interprets the disjunctive rule head operator $\mid$ differently from the classical connective $\vee$. Let $\mathcal{H}_{1}=E_{1}|\cdots|$ $E_{k}$ and $\mathcal{H}_{2}=E_{1} \vee \cdots \vee E_{k}$ be two rule heads and let $I$ be an interpretation that satisfies $\mathcal{H}_{2}$. Then there may be up to $k$ head selection functions for $\mathcal{H}_{1}$, each selecting one alternative $E_{i}$ that is satisfied by $I$, which leads to at most $k$ disjunctive program reducts; in contrast, there is only one head selection function for $\mathcal{H}_{2}$, i.e., $\operatorname{sel}\left(\mathcal{H}_{2}, I\right)=\mathcal{H}_{2}$, which leads to only one disjunctive program reduct. Different reducts may lead to different candidate answer sets and thus disjunctive programs with rule heads like $\mathcal{H}_{1}$ are different from programs with rule heads like $\mathcal{H}_{2}$.

Moreover, the above semantics does not require that answer sets should be minimal models; it only requires answer sets to be minimal among all candidate answer sets.

In order to stress the intuition that candidate answer sets are determined by means of a chosen head selection function for applying rules $H_{1}|\cdots| H_{k} \leftarrow \operatorname{Body}$, where one alternative $H_{i}$ from the head is inferred when Body is satisfied, we refer to the above answer set semantics as determining inference (DI) semantics for disjunctive programs; we call answer sets of DI-semantics DI-answer sets and models satisfying condition (1) of Definition 5 candidate DI-answer sets.

\section{DI-Semantics for Simple Disjunctive Programs}

By replacing the base semantics $\mathcal{X}$ in Definition 5 with $\mathrm{GL}_{n l p}$-semantics we induce a DI-answer set semantics for simple disjunctive programs.

Definition 6. A model $I$ of a simple disjunctive program $\Pi$ is a DI-answer set of $\Pi$, if (1) for some head selection function sel, $I$ is an answer set of $\Pi_{s e l}^{I}$ under $\mathrm{GL}_{n l p}$-semantics, and (2) $\Pi$ has no model $J \subset I$ satisfying condition (1).

A DI-answer set is not necessarily a GL-answer set, but for simple normal programs and positive simple disjunctive programs, DI-semantics agrees with GL-semantics.

Theorem 1. Let $\Pi$ be a simple normal program or a positive simple disjunctive program. Then an interpretation I is a DIanswer set of $\Pi$ iff $I$ is a GL-answer set of $\Pi$.

It is particularly interesting to observe that GL-semantics can also be characterized using the disjunctive program reduct $\Pi_{\text {sel }}^{I}$ of Definition 4 simply by requiring that for every (instead of some) head selection function sel, $I$ is an answer set of $\Pi_{s e l}^{I}$ under $\mathrm{GL}_{n l p}$-semantics. This reveals the essential difference between DI-semantics and GL-semantics.

Theorem 2. A model I of a simple disjunctive program $\Pi$ is a GL-answer set of $\Pi$ iff for every head selection function sel, $I$ is an answer set of $\Pi_{\text {sel }}^{I}$ under $G L_{n l p}$-semantics.

As GL-answer sets of a simple disjunctive program $\Pi$ are minimal models of $\Pi$, the following corollary is immediate.
Corollary 1. Let $\Pi$ be a simple disjunctive program. If $I$ is a GL-answer set, then I is a DI-answer set.

\section{DI-Semantics for General Programs}

General normal programs consist of rules of the form $H \leftarrow$ $B$, where $H$ and $B$ are first-order formulas. To overcome the problem of circular justifications with those answer set semantics for general normal programs such as those in [Pearce, 2006; Truszczynski, 2010; Bartholomew et al., 2011; Faber et al., 2011; Ferraris et al., 2011] based on classical logic, [Shen et al., 2014] presented the well-justified semantics whose answer sets have a level mapping and thus are free of circular justifications, in analogy to the level mapping of $\mathrm{GL}_{n l p}{ }^{-}$ semantics for simple normal programs [Fages, 1994]. [Shen et al., 2014] left extending the well-justified semantics to general disjunctive programs as an open problem; we can elegantly close it by replacing the base semantics $\mathcal{X}$ in Definition 5 with the well-justified semantics.

The well-justified semantics is based on the one-step provability operator $T_{\Pi}(O, N)$, which extends the well-known immediate consequence operator [van Emden and Kowalski, 1976] from Horn programs to general normal programs.

Definition 7 ([Shen et al., 2014]). Let $\Pi$ be a general normal program, and let $O$ and $N$ be two first-order theories. Then

$T_{\Pi}(O, N)=\{\operatorname{head}(r) \mid r \in \operatorname{ground}(\Pi), O \cup N \models \operatorname{body}(r)\}$.

Informally, $T_{\Pi}(O, N)$ collects all heads of grounded rules whose bodies are entailed by $O \cup N$. For fixed $N$, the entailment $\models$ is monotone in $O$, so $T_{\Pi}(O, N)$ is monotone w.r.t. $O$, i.e., for any theories $O_{1} \subseteq O_{2}$, we have $T_{\Pi}\left(O_{1}, N\right) \subseteq$ $T_{\Pi}\left(O_{2}, N\right)$. As moreover $T_{\Pi}(O, N)$ is finitary, the inference sequence $\left\langle T_{\Pi}^{i}(\emptyset, N)\right\rangle_{i=0}^{\infty}$, where $T_{\Pi}^{0}(\emptyset, N)=\emptyset$ and for $i \geq 0$ $T_{\Pi}^{i+1}(\emptyset, N)=T_{\Pi}\left(T_{\Pi}^{i}(\emptyset, N), N\right)$, will converge to a least fixpoint, denoted $\operatorname{lfp}\left(T_{\Pi}(\emptyset, N)\right)$.

The well-justified (WJ) semantics is then defined in terms of $\operatorname{lfp}\left(T_{\Pi}\left(\emptyset, \neg I^{-}\right)\right)$, i.e., derivability under the closed-world assumption applied to candidate answer $I$, as follows.

Definition 8 ([Shen et al., 2014]). Let $I$ be a model of a general normal program $\Pi$. Then $I$ is a $W J$-answer set of $\Pi$ if lfp $\left(T_{\Pi}\left(\emptyset, \neg I^{-}\right)\right) \cup \neg I^{-} \models A$ for every $A \in I$.

By replacing $\mathcal{X}$ in Definition 5 with $\mathrm{WJ}$-semantics we induce a DI-answer set semantics for general programs.

Definition 9. A model $I$ of a general disjunctive program $\Pi$ is a DI-answer set of $\Pi$ if (1) for some head selection function sel, $I$ is a WJ-answer set of $\Pi_{\text {sel }}^{I}$, and (2) $\Pi$ has no model $J \subset I$ satisfying condition (1).

Intuitively, a DI-answer set is a model that is minimal among all models that can be nondeterministically (by means of a head selection function) inferred by iteratively applying rules via a bottom up fixpoint sequence.

Corollary 2. For a general normal program, I is a DIanswer set iff I is a WJ-answer set. For a simple disjunctive program, I is a DI-answer set under Definition 9 iff $I$ is a DI-answer set under Definition 6. 


\section{Computational Complexity}

We address the computational complexity of propositional logic programs, where we focus on the DI-semantics with the well-justified semantics [Shen et al., 2014] as the base semantics and refer to it as DI-WJ answer set semantics.

Theorem 3. Given a propositional simple (resp. general) disjunctive program $\Pi$ and a ground literal $L$, deciding whether (i) П has some DI-WJ answer set is NP-complete (resp. $\Sigma_{2}^{p}$ complete), (ii) $L$ is true in every DI-WJ answer set of $\Pi$ is $\Pi_{2}^{p}$-complete (resp. $\Pi_{3}^{p}$-complete), and (iii) $L$ is true in some DI-WJ answer set of $\Pi$ is $\Sigma_{2}^{p}$-complete (resp. $\Sigma_{3}^{p}$-complete).

Analogous results hold for other semantics such as FLPsemantics [Faber et al., 2011]. Compared to GL-/FLPsemantics, the complexity of brave and cautious reasoning increases under DI-semantics by one level of $\mathrm{PH}$, thus offering higher problem solving capacity. Computing a DI-WJ answer set is complete for the NP- resp. $\Sigma_{2}^{p}$-functions and feasible with bounded many witness oracle calls [Buss et al., 1993; Janota and Marques-Silva, 2016] in polynomial time.

\section{Difference between Disjunctive Rule Heads and Choice Constructs}

Like disjunctive rule heads, choice constructs [Simons et al., 2002; Ferraris and Lifschitz, 2005; Calimeri et al., 2012] are also used to express a set of alternatives. However, a disjunctive rule head $a_{1}|\cdots| a_{m}$ and a choice construct of the form $u_{1}\left\{a_{1}, \cdots, a_{m}\right\} u_{2}$, where $m>0,0 \leq u_{1} \leq u_{2} \leq m$, and the $a_{i}$ 's are ground atoms, are essentially different.

Let $\alpha=\left\{a_{1}, \cdots, a_{m}\right\}$ and $\beta=\left\{\gamma \mid \gamma \subseteq \alpha\right.$ and $u_{1} \leq$ $\left.|\gamma| \leq u_{2}\right\}$. The choice construct $u_{1}\left\{a_{1}, \cdots, a_{m}\right\} u_{2}$ says that any $\gamma \in \beta$ can be chosen as answer.

For a logic program $\Pi$, let $A S(\Pi)$ denote the set of answer sets of $\Pi$. Let $\Pi^{\prime}$ be $\Pi$ extended with a choice construct $u_{1}\left\{a_{1}, \cdots, a_{m}\right\} u_{2}$. Then the set of answer sets of $\Pi^{\prime}$ is

$$
A S\left(\Pi^{\prime}\right)=\bigcup_{\gamma \in \beta} A S(\Pi \cup\{a \mid a \in \gamma\} \cup\{\neg b \mid b \in(\alpha \backslash \gamma)\}) \text {. }
$$

Example 2. Let $\Pi=\{b\}$ and $\Pi^{\prime}=\Pi \cup\{1\{a, b\} 2\}$. Then $A S\left(\Pi^{\prime}\right)=A S(\Pi \cup\{a, \neg b\}) \cup A S(\Pi \cup\{\neg a, b\}) \cup A S(\Pi \cup$ $\{a, b\})$. $\Pi \cup\{a, \neg b\}$ has no model and thus no answer set, $\Pi \cup\{\neg a, b\}$ has a single answer set $\{b\}$, and $\Pi \cup\{a, b\}$ has a single answer set $\{a, b\}$. Therefore, $\Pi^{\prime}$ has in total two answer sets, $\{b\}$ and $\{a, b\}$.

A disjunctive rule head $a_{1}|\cdots| a_{m}$ infers one atom $a_{i}$ from $\alpha$ and differs essentially from a choice construct $1\left\{a_{1}, \cdots, a_{m}\right\} u$. When $u=1$, the choice construct $1\left\{a_{1}\right.$, $\left.\cdots, a_{m}\right\} 1$ in a logic program $\Pi$ enforces every answer set of $\Pi$ to contain exactly one $a_{i}$ from $\alpha$. In contrast, though $a_{1}|\cdots| a_{m}$ infers only one $a_{i}$ from $\alpha$, a DI-answer set may contain other atoms $a_{j} \in \alpha$, which are inferred by other rules in a disjunctive program. When $u>1$, the choice construct $1\left\{a_{1}, \cdots, a_{m}\right\} u$ allows for answer sets $I$ and $J$ with $I \subset J$. This will not happen with $a_{1}|\cdots| a_{m}$ for DI-answer sets.

\section{Relation to Split and Fork Programs}

For simple disjunctive programs $\Pi$, [Hitzler and Seda, 1999] proposed to split $\Pi$ into a collection of simple normal programs, called normal derivatives $P(\Pi)$, which informally are obtained from ground $(\Pi)$ by replacing every rule $A_{1}|\cdots| A_{k} \leftarrow \operatorname{bod} y(r), k \geq 2$, arbitrarily with one or more rules $A_{i} \leftarrow \operatorname{body}(r), 1 \leq i \leq k$. E.g., $\Pi=\{p \mid q \leftarrow \neg s\}$ has three normal derivatives: $P_{1}(\Pi)=\{p \leftarrow \neg s\}, P_{2}(\Pi)=$ $\{q \leftarrow \neg s\}$, and $P_{3}(\Pi)=\{p \leftarrow \neg s, q \leftarrow \neg s\}$.

[Hitzler and Seda, 1999] aimed to use normal derivatives to characterize GL-semantics of a simple disjunctive program $\Pi$. They showed that every answer set of $\Pi$ under GLsemantics is an answer set of some normal derivative of $\Pi$ under $\mathrm{GL}_{n l p}$-semantics. E.g. for the program $\Pi$ from above, $I=\{p\}$ is an answer set of $\Pi$ under GL-semantics and an answer set of $P_{1}(\Pi)$ under $\mathrm{GL}_{n l p}$-semantics. However, they left a precise characterization open, stated as the problem to determine for every interpretation $I$ some normal derivatives such that $I$ is an answer set of $\Pi$ under GL-semantics iff $I$ is an answer set of these normal derivatives under $\mathrm{GL}_{n l p}$-semantics.

The characterization of GL-semantics by the disjunctive program reduct (Theorem 2) enables us to provide a solution for this open problem. For a logic program $\Pi$, an interpretation $I$ and a head selection sel on $I$, let

$P_{\text {sel }}(\Pi, I)=\{\operatorname{sel}(\operatorname{head}(r), I) \leftarrow \operatorname{body}(r) \mid r \in \operatorname{ground}(\Pi)\}$, $N D(\Pi, I)=\left\{P_{\text {sel }}(\Pi, I) \mid\right.$ sel is a head selection on $\left.I\right\}$.

Note that $N D(\Pi, I)$ is the collection of normal derivatives obtained by applying every head selection on $I$. Thus for any head selection sel on a model $I$, we have

$$
\begin{array}{r}
P_{\text {sel }}(\Pi, I)=\{\operatorname{sel}(\operatorname{head}(r), I) \leftarrow \operatorname{body}(r) \mid r \in \operatorname{ground}(\Pi)\} \\
=\Pi_{\text {sel }}^{I} \cup\{\operatorname{sel}(\operatorname{head}(r), I) \leftarrow \operatorname{bod}(r) \mid r \in \operatorname{ground}(\Pi) \\
\text { and } \operatorname{body}(r) \text { is not satisfied by } I\} .
\end{array}
$$

A solution to the above open problem is then as follows.

Theorem 4. An interpretation I is an answer set of a simple disjunctive program $\Pi$ under GL-semantics iff $I$ is an answer set of every $P(\Pi) \in N D(\Pi, I)$ under $G L_{n l p}$-semantics.

In independent work and parallel to ours, [Aguado et al., 2019] proposed a new construct "|" for answer programs called fork, which aims at overcoming problems with omitting auxiliary atoms in choice constructs. Informally, under fork semantics the answer sets of $\{E \mid F\} \cup \Pi$ are the answer sets of $\{E\} \cup \Pi$ plus the answer sets of $\{F\} \cup \Pi$. Accordingly, $P=\{a|b, b| c\}$ has the fork-answer sets $\{a, b\},\{a, c\},\{b\}$ and $\{b, c\}$, while its DI-answer sets are $\{a, c\}$ and $\{b\}$. They diverge as DI-semantics operates in a sense globally on alternatives in different rules (by item (2) in Definition 5), while fork semantics operates locally treating them independently.

Notably, selection functions similar to ours were used in [Vennekens et al., 2004] to define probabilistic semantics for logic programs with annotated disjunctions. However, they do not depend on an interpretation and result (disregarding probabilities) in all normal derivatives picking always a single rule $A_{i} \leftarrow$ body (in the example, $P_{1}(\Pi)$ and $P_{2}(\Pi)$ ). Thus like fork semantics, this semantics has a local flavor.

\section{Acknowledgments}

This work has been supported in part by NSFC grants 61976205 and 61379043, and the Austrian Science Fund (FWF) grant P27730. 


\section{References}

[Aguado et al., 2019] Felicidad Aguado, Pedro Cabalar, Jorge Fandinno, David Pearce, Gilberto Pérez, and Concepción Vidal. Forgetting auxiliary atoms in forks. Artif. Intell., 275:575-601, 2019.

[Bartholomew et al., 2011] M. Bartholomew, J. Lee, and Y. Meng. First-order extension of the FLP stable model semantics via modified circumscription. In Proc. 22nd Int'l Joint Conference on Artificial Intelligence (IJCAI11), pages 724-730, 2011.

[Buss et al., 1993] Samuel Buss, Jan Krajǐček, and Gaisi Takeuti. On provably total functions in bounded arithmetic theories. In Peter Clote and Jan Krajiček, editors, Arithmetic, Proof Theory and Computational Complexity, pages 116-61. Oxford University Press, 1993.

[Calimeri et al., 2012] Francesco Calimeri, Wolfgang Faber, Martin Gebser, Giovambattista Ianni, Roland Kaminski, Thomas Krennwallner, Nicola Leone, Francesco Ricca, and Torsten Schaub. ASP-Core-2: Input language format, 2012. https://www.mat.unical.it/aspcomp2013/files/ ASP-CORE-2.01c.pdf.

[Eiter and Gottlob, 1995] T. Eiter and G. Gottlob. On the computational cost of disjunctive logic programming: Propositional case. Annals of Mathematics and Artificial Intelligence, 15(3-4):289-323, 1995.

[Faber et al., 2011] W. Faber, G. Pfeifer, and N. Leone. Semantics and complexity of recursive aggregates in answer set programming. Artificial Intelligence, 175(1):278-298, 2011.

[Fages, 1994] François Fages. Consistency of clark's completion and existence of stable models. Journal of Methods of Logic in Computer Science, 1:51-60, 1994.

[Ferraris and Lifschitz, 2005] Paolo Ferraris and Vladimir Lifschitz. Mathematical foundations of answer set programming. In Sergei N. Artëmov, Howard Barringer, Artur S. d'Avila Garcez, Luís C. Lamb, and John Woods, editors, We Will Show Them! Essays in Honour of Dov Gabbay, Volume One, pages 615-664. College Publications, 2005.

[Ferraris et al., 2011] P. Ferraris, J. Lee, and V. Lifschitz. Stable models and circumscription. Artificial Intelligence, 175(1):236-263, 2011.

[Gelfond and Lifschitz, 1988] Michael Gelfond and Vladimir Lifschitz. The stable model semantics for logic programming. In Logic Programming, Proceedings of the Fifth International Conference and Symposium, pages 1070-1080, 1988.

[Gelfond and Lifschitz, 1991] M. Gelfond and V. Lifschitz. Classical negation in logic programs and disjunctive databases. New Generation Computing, 9:365-385, 1991.

[Hitzler and Seda, 1999] Pascal Hitzler and Anthony Karel Seda. Multivalued mappings, fixed-point theorems and disjunctive databases. In Proceedings of the 3rd Irish Conference on Formal Methods, Galway, Eire, pages 113-131. British Computer Society, 1999.
[Janota and Marques-Silva, 2016] Mikolás Janota and Joao Marques-Silva. On the query complexity of selecting minimal sets for monotone predicates. Artif. Intell., 233:7383, 2016.

[Pearce, 2006] D. Pearce. Equilibrium logic. Annals of Mathematics and Artificial Intelligence, 47(1-2):3-41, 2006.

[Shen and Eiter, 2019] Y. D. Shen and T. Eiter. Determining inference semantics for disjunctive logic programs. Artificial Intelligence, 277:1-28, 2019.

[Shen et al., 2014] Y. D. Shen, K. Wang, T. Eiter, M. Fink, C. Redl, T. Krennwallner, and J. Deng. FLP answer set semantics without circular justifications for general logic programs. Artificial Intelligence, 213:1-41, 2014.

[Simons et al., 2002] P. Simons, I. Niemela, and T. Soininen. Extending and implementing the stable model semantics. Artificial Intelligence, 138(1-2):181-234, 2002.

[Truszczynski, 2010] M. Truszczynski. Reducts of propositional theories, satisfiability relations, and generalizations of semantics of logic programs. Artificial Intelligence, 174(16-17):1285-1306, 2010.

[van Emden and Kowalski, 1976] M. H. van Emden and R. A. Kowalski. The semantics of predicate logic as a programming language. Journal of the ACM, 23(4):733-742, 1976.

[Vennekens et al., 2004] Joost Vennekens, Sofie Verbaeten, and Maurice Bruynooghe. Logic programs with annotated disjunctions. In Bart Demoen and Vladimir Lifschitz, editors, Logic Programming, 20th International Conference, ICLP 2004, Saint-Malo, France, September 6-10, 2004, Proceedings, volume 3132 of Lecture Notes in Computer Science, pages 431-445. Springer, 2004. 